\title{
Disputas en torno a los planes de cuidado en la internación domiciliaria: una reflexión necesaria
}

\author{
The debate over care plans in home medical care: \\ a necessary reflection
}

Luís Claudio de Carvalho ${ }^{1}$

Laura Camargo Macruz Feuerwerker ${ }^{2}$

Emerson Elias Merhy 3

${ }^{1}$ Médico, Universidad

Federal Fluminense. Magíster en Clínica Médica, Universidad Federal de Río de Janeiro (UFRJ). Integrante de la línea de investigación Micropolítica del Trabajo y el Cuidado en Salud del programa de posgrado del Departamento de Clínica Médica, UFRJ, Brasil. lucacarv@uol.com.br

2 Médica, Universidad de San Pablo (USP). Doctora en Salud Pública, USP.

Profesora de la Universidad Fluminense. Investigadora de la línea de investigación Micropolítica del Trabajo y el Cuidado en Salud del programa de posgrado del Departamento de Clínica Médica, Universidad Federal de Río de Janeiro, Brasil. laura.macruz@gmail.com

3 Médico, Universidad de San Pablo. Libre-Docente, Universidad del Estado de Campinas. Profesor del Posgrado del Departamento de Clínica Médica,

Universidad Federal de Río de Janeiro. Coordinador de la línea de investigación Micropolítica del Trabajo y el Cuidado en Salud, Brasil. emerson.merhy@gmail.com
RESUMEN La Atención Domiciliaria se presenta en Brasil como una modalidad de atención a la salud con potencial substitutivo y capacidad de ofrecer cuidado con calidad, garantizando a la familia y al usuario la oportunidad de manejar salud y enfermedad desde una óptica innovadora. Este artículo presenta una reflexión sobre el analizador -disputa de planes de cuidado- identificado en uno de los estudios de caso de la investigación sobre implantación de la atención domiciliaria en el Sistema Único de Salud (SUS). El foco del análisis fue la micropolítica del trabajo en salud en la producción del cuidado, involucrando al equipo y a las/los cuidadores.

PALABRAS CLAVE Servicios de Atención de Salud a Domicilio; Trabajo; Cuidadores; Gestión en Salud; Prestación de Atención de Salud.

ABSTRACT In Brazil, home medical care emerges as a potential alternative that offers quality care, ensuring relatives and patients the opportunity to handle health and disease from an innovative perspective. This article provides a reflection on the analyzer -debate over care plans- identified in one of the case studies on home medical care implementation's investigation within the Single Health Care System (SUS). The analysis focuses on the micro policy of health care work in care production, involving both the team and carers.

KEY WORDS Home Care Services; Work; Caregivers; Health Management; Delivery of Health Care. 


\section{INTRODUCCIÓN}

En el campo de las prácticas de salud (1) -a pesar de haber distintas comprensiones sobre su naturaleza, como intervención sobre el cuerpo biológico socialmente investido (2), como lugar de búsqueda de la cura (3) o como actos productores de cuidado individual o colectivo $(4,5,6)$ - hay una construcción de intervenciones tecnológicamente orientadas que buscan actuar a partir de un lugar socialmente legitimado, como es el caso de la medicina y de la salud pública, que se traducen en acciones sobre un otro, como objeto, en búsqueda de ciertas finalidades que no necesariamente son las mismas sino que dependen del contexto histórico y social en el cual esas prácticas se realizan.

Sin embargo, existe un elemento de tensión en este campo de producción de actos de salud que ha sido poco explorado en los estudios que analizan la manera en que se conforman los procesos del cuidado en salud. Ese elemento se refiere al hecho de que estos cuidados son siempre un proceso en el cual un otro, como objeto, es el blanco de las acciones, pero no necesariamente es un ser inerte; por el contrario, sistemáticamente en las experiencias cotidianas de producción del cuidado se percibe cuánto este objeto-blanco de las intervenciones en salud es efectivamente un sujeto en acción (4,7-9).

Esa situación muchas veces es percibida por los trabajadores de salud como la capacidad del usuario de resistir a un acto de cuidado profesional, como si estuviera inventando su modo de cuidarse. Eso generalmente es visto como algo negativo por parte de los distintos profesionales de salud. Sin embargo, es posible reconocer este proceso como más común de lo que parece, o también como constitutivo de cualquier momento relacional del cuidado en salud, en cualquier lugar donde ocurra.

Se destaca que el cuidado aquí referido no es entendido como un nivel de atención del sistema, ni como un procedimiento simplificado, sino como una acción integral, que tiene significados y sentidos volcados hacia la comprensión de la salud como derecho (6). La acción integral es también entendida como la "interrelación" de las personas (9), o sea, la acción integral como efectos y repercusiones de interacciones positivas entre usuarios, profesionales e instituciones, que es traducida en actitudes como: tratamiento digno respetuoso, de calidad, protección y vínculo. El cuidado se traduce también en la elección ética y política de las tecnologías a ser utilizadas en la resolución de los problemas de salud $(4,5)$.

En este texto, a través de un estudio realizado en el campo de la internación domiciliaria, se parte de la evidencia de ese otro, objetoblanco, como sujeto actuante, en un proceso efectivo de disputa por el cuidado en salud. No se considera esto como un momento negativo de resistencia, sino como parte fundamental de la construcción de los planes terapéuticos. A partir de allí, se sacan algunas consecuencias para pensar los modelos de atención.

El proyecto de investigación citado anteriormente tuvo como objetivo evaluar las modalidades existentes de "Atención Domiciliaria y proponer modelos posibles de financiamiento, regulación, evaluación y control, particularmente frente a los usuarios con dependencia funcional, candidatos a intervenciones terapéuticas que aseguren mayores niveles de autonomía, y alivio del sufrimiento" (a).

El trabajo de campo nos permitió percibir una diversidad de experiencias en todo el país, siendo posible identificar analizadores comunes y específicos (10) a lo largo del desarrollo de la investigación.

En este artículo se pretende puntualizar la disputa del plan de cuidado, a partir de una de las experiencias estudiadas en la ciudad de Río de Janeiro.

\section{METODOLOGÍA}

En esta investigación se desarrollaron las siguientes etapas:

1. Mapeo de las experiencias de atención domiciliaria, para identificar los programas de atención domiciliaria conducidos de manera independiente por las tres esferas de gestión en salud (municipal, provincial y nacional) en el ámbito del Sistema Único de Salud (SUS). 
2. Trabajo de campo para el reconocimiento y análisis del servicio focalizado, mediante la aplicación del "kit investigador" compuesto por: carta de presentación; términos de consentimiento libre y esclarecido -modelo para los profesionales de la salud y otro para los usuarios y familiares; guía de entrevista para el gestor del servicio de atención domiciliaria (han sido analizados todos los servicios existentes).

$\rightarrow$ La guía de entrevista fue estructurada tomando como referencia los analizadores artificiales (b), definidos por el equipo de investigación [modos de organización y composición del equipo; articulación del Programa con el sistema local de salud; características del cuidador; la conformación del acto de cuidar; racionalidad (efectividad, eficiencia y eficacia); evaluación del Programa (herramientas de evaluación)]. Además, también fue utilizado el "Diario de Campo" para la recolección de datos (11).

3. Estudio de casos trazadores.

$\rightarrow$ El caso trazador es un método que permite evaluar el proceso de trabajo de un equipo de salud a partir de la reconstitución de la trayectoria de un caso, desde distintos puntos de vista y a partir de distintas fuentes, posibilitando un mejor análisis del cuidado prestado al usuario (12).

$\rightarrow$ La lógica de construcción del caso trazador se basó en la herramienta "flujograma analizador" (4) con el objetivo de observar más atentamente la micropolítica del trabajo en salud en la producción del cuidado. Para ello fue necesario entrevistar a los trabajadores de salud, al cuidador/a y al usuario involucrados en la conducción del caso. Por medio de sus palabras se reconstruyó el camino recorrido por el usuario y sus momentos de intercomunicación con los otros actores. Las entrevistas con los profesionales han sido realizadas en la unidad de salud y con el/la cuidador(a) y el usuario en el domicilio. En cada ciudad, se ha construido un banco de casos trazadores y los más significativos (elegidos en conjunto con los equipos locales) han sido analizados. $\rightarrow$ Vale destacar que se ha optado por esta herramienta, dado que ha demostrado ser efectiva en diversas experiencias $(4,8,13$ 19). Es un instrumento potente para la construcción de mapas de procesos de trabajo de un servicio de salud y de su articulación con la red.

$\rightarrow$ Luego del procesamiento del material recolectado, se realizó la devolución hacia el equipo, a través de un flujograma, posibilitando que ellos se interrogaran respecto al proceso de trabajo existente en el servicio y en la red de servicios de salud. De este modo fue posible promover un análisis más específico de las relaciones establecidas entre los trabajadores y entre ellos y el usuario, además de la percepción sobre las tecnologías utilizadas, sus manejos y los problemas del proceso de trabajo del equipo.

\section{EL CASO DEL NIÑO FELIZ}

A continuación, se presenta un breve relato de un caso, útil para posibilitar una reflexión sobre la micropolítica del trabajo en salud.

El caso seleccionado para la elaboración del flujograma analizador fue el de un niño de nueve años Ilamado Feliz (c), que estuvo internado en un hospital durante cuatro años debido a un cuadro de neumonía viral que evolucionó a una Enfermedad Pulmonar Obstructiva Crónica (EPOC).

Su primera internación hospitalaria ocurrió a los nueve meses de edad y su ingreso en el Programa de Atención Domiciliaria (PAD) del mismo hospital ocurrió a partir de los cuatro años y medio de edad. Sin embargo, para que el niño pudiera retornar a su casa fue necesario que se reconstruyera el domicilio de su familia (durante ese proceso, la familia había estado viviendo en la casa de parientes durante un año).

De regreso a su casa, Feliz necesitó de la utilización de algunos equipos (concentrador de $\mathrm{O}_{2}$, BiPAP y cilindro de $\mathrm{O}_{2}$ ), además de la presencia regular del equipo del Programa de Atención Domiciliaria (PAD) en su casa. El equipo está compuesto por la trabajadora social, fisioterapeutas, médico, enfermera, técnica de enfermería y psicóloga. 
El momento del regreso fue de gran alegría, tanto para Feliz como para la familia (representada por la madre, dos hermanas, un sobrino y el padrastro). La madre asume entonces el papel de cuidadora, es quien se encarga de ejecutar el plan de cuidado elaborado por el equipo para el niño.

A lo largo de la internación domiciliaria, la madre dejó de cumplir integralmente la conducta prescrita por el equipo, produciendo cierta incomodidad en los profesionales, que reaccionaron proponiendo estrategias con el objetivo de lograr que la cuidadora cumpliera lo esperado/programado.

Durante las entrevistas con los involucrados fue posible percibir que la cuidadora no estaba totalmente de acuerdo con el plan de cuidados propuesto por el equipo; ella tenía un plan propio. Por ejemplo, la madre permitía que el niño jugara con otros niños sin hacer uso continuo del oxígeno; le permitía remontar barriletes, jugar en la arena, etc. Tales acciones no eran aprobadas por el equipo, quienes consideraban que el niño debía estar las 24 horas ligado al oxígeno. Durante nuestra observación afloró un importante analizador natural del caso (d) -la disputa sobre los planes de cuidado entre la cuidadora y el equipo- que provocó una serie de indagaciones respecto a la producción del cuidado: ¿el equipo no debería ser más flexible en la elaboración del plan? ¿Por qué la cuidadora no podía participar de la construcción del plan? ¿Es posible compatibilizar los dos planes de cuidado? ¿Cuáles son los planes de cuidado en disputa?

\section{REFLEXIONANDO SOBRE EL ANALIZADOR DISPUTA DE PLANES DE CUIDADO}

El caso de Feliz nos posibilita reflexionar sobre diversos aspectos, entre ellos, la racionalidad que orienta las acciones de los actores clave. El equipo trabaja sobre todo con un saber técnico-científico focalizado en la enfermedad, mientras que la cuidadora trabaja con un saber de madre y cuidadora que está direccionado a la producción de vida del niño, acarreando los distintos planes de cuidado.
Se percibe que la madre, al mirar a Feliz, no observa un paciente sino a su hijo, que necesita cuidado y también vivir como un niño. Sin embargo, el equipo, siguiendo la racionalidad de la "clínica moderna, orientada por la muerte (anatomía patológica), se asienta en la causa de la enfermedad (agente patogénico) y en su origen espacio-temporal (localización orgánica e historia sintomática)" (3), teniendo como objetivo el control/cura de la enfermedad.

Esa distinción propicia varias disputas y conflictos entre los actores, siendo que el saber que trae la cuidadora no es legitimado por el modelo médico hegemónico $y$, consecuentemente, por el equipo del PAD.

¿Qué hacer frente a esa situación? Reflexionamos sobre la posibilidad de una nueva racionalidad que nos trae la figura del cuidador, focalizada en la producción de vida y en las necesidades de salud de los usuarios.

Es importante, en este caso, recuperar el concepto de necesidades de salud tal como es desarrollado por Cecílio (20).

Según el autor, las necesidades de salud pueden ser clasificadas en cuatro conjuntos:

1. Tener "buenas condiciones de vida", entendiéndose que el modo de vida produce diferentes necesidades de salud.

2. Tener acceso y poder consumir toda la tecnología de salud capaz de mejorar y prolongar la vida.

3. Desarrollar vínculos (a)efectivos entre cada usuario y un equipo y/o profesional, vínculo comprendido como referencia y relación de confianza, algo así como "la cara" del sistema de salud para el usuario.

4. Garantizar que "cada persona pueda desarroIlar mayores grados de autonomía en su modo de llevar la vida".

Trazando un paralelo con los conceptos formulados por Cecílio (20), se percibe que el equipo no consigue garantizar el primero y el cuarto ítem, presentados por el autor, mientras la madre consigue contemplar todos los ítems, aunque no plenamente.

Otros puntos que merecen ser destacados son las tecnologías utilizadas en el trabajo en salud desarrollado por el equipo, y las utilizadas 
por la cuidadora considerando las racionalidades identificadas.

En la literatura, la producción sobre tecnologías en salud viene incrementándose hace más de una década, y fue iniciada por Gonçalves (2), que presentó la distinción entre las tecnologías que están inscritas en las máquinas y en los instrumentos de trabajo y aquellas relacionadas al conocimiento técnico. El autor denominó a las primeras como "tecnologías materiales" y a las otras como "tecnologías no materiales". La novedad de su proposición es construir la comprensión del conocimiento como tecnología. O sea, los profesionales pasan a ser comprendidos como sujetos sociales portadores de conocimiento y capacidad de ofertar una asistencia de calidad.

De ese modo, el trabajo humano se presenta como fundamental e insustituible en la producción del cuidado. Ya las máquinas e instrumentos (tecnologías materiales) deben cumplir el papel de tecnologías auxiliares en el trabajo en salud, posibilitando la definición del diagnóstico y de las terapias (18).

Dando continuidad a los estudios sobre el tema, Merhy $(4,5)$ emplea una metáfora para mostrar cómo el uso de las tecnologías puede impactar en el trabajo en salud, expresando, incluso, el modelo asistencial subyacente. Él utiliza la "imagen" de tres maletines para demostrar didácticamente el arsenal tecnológico de cada profesional de salud, lo que no significa una mirada compartimentada, pues los tres tipos de tecnologías expresan procesos productivos singulares:

$\rightarrow$ el primer maletín representa los instrumentos y equipamientos (tecnologías duras);

$\rightarrow$ el segundo, los saberes estructurados, como la clínica y la epidemiología (tecnologías blandas-duras);

$\rightarrow$ el tercero, el espacio relacional trabajadorusuario (tecnologías blandas), espacio de relaciones entre sujetos, que solamente tiene materialidad en el acto, como es el caso de los procesos de protección, vínculo y responsabilización $(4,21-24)$ considerados como prácticas de intersección en salud (25).

Lo que el autor quiere resaltar con estos ejemplos es que las combinaciones entre los tres "maletines" son estratégicas, definidoras del sentido de los modelos de atención a la salud (5).

En este caso se puede verificar que los profesionales de salud operan con tecnologías duras (equipamientos y protocolos clínicos), blandas-duras (saber profesional) y blandas (vínculo). Sin embargo, siguiendo la lógica de la medicina moderna, los profesionales utilizan las tecnologías duras como guía en la producción del cuidado, no habiendo posibilidad de escucha y de valoración de la necesidad de Feliz de jugar e interactuar con otros niños.

Observando a la cuidadora, se percibe que ella opera con otra combinación de tecnologías: dura (manipulación de los equipos y medicamentos), blanda-dura (saber de cuidadora y madre) y blanda (vínculo-responsabilización, protección, afecto, amistad, compañerismo, etc.). En su caso, siguiendo la lógica de la producción de vida, son las tecnologías blandas las que operan como guía de la producción del cuidado prestado a Feliz, haciendo posible la atención a sus necesidades.

Al observar el trabajo en salud desarroIlado por este equipo, podemos percibir que es el médico quien define el plan de cuidado y los demás profesionales generalmente ejecutan lo pautado. Pero la cuidadora impone su ritmo, atendiendo a las necesidades propias y las del niño, interfiriendo activamente en la cadena productiva del cuidado, siendo muchas veces mirada por el equipo como alguien que no se ha adherido al plan, como alguien irresponsable y que no cuida.

Es válido recordar que el desarrollo de la "medicina científica" influyó sobre las demás profesiones de la salud, basado en una perspectiva biologicista y mecanicista del ser humano, en la cual el cuerpo es mirado como una máquina, elemento esencial del modo de producción capitalista. De este modo, la mediación entre el hombre y las enfermedades -realizada por los profesionales de la salud- pasó darse cada vez más a partir de la creciente incorporación de procedimientos y equipamientos que permitirían el "reparo" de los daños.

Utilizando una representación de Merhy (5), la medicina tecnológica es la expresión de actos fragmentados sobre un usuarioinsumo, donde el vínculo pasa a estar con el examen, con la consulta y no con el usuario. 
Al contrario de lo hegemónico, un modelo pautado en el mundo de las necesidades de los usuarios estará centrado en las tecnologías blandas y blandas-duras, configurando un proceso de trabajo en el cual el aspecto relacional (de intersección) tiene una presencia fuerte (5). Por lo tanto, en las tecnologías blandas, que están presentes en el trabajo de todos los profesionales de la salud, las prácticas de protección, vínculo y responsabilización son una apuesta anti-hegemónica de alternativa a las prácticas centradas en el núcleo duro de las tecnologías y no en el cuidado.

El caso presentado posibilita constatar que hay diferencias en la producción del cuidado, como ya fue descrito anteriormente. En el cuidado prestado por la cuidadora, la tecnología blanda comanda el proceso de trabajo y el medicamento y los equipamientos operan como auxiliares. La relación entre cuidadora y usuario es dinámica, hay escucha, percepción de la singularidad, valorización de los movimientos mutuos de cuidado y producción de salud.

Ya en el cuidado prestado por el equipo, se puede decir que el proceso de trabajo es comandado por la tecnología dura, materializada en los equipamientos y procedimientos y en la tecnología blanda-dura, materializada en el saber estructurado de los profesionales. De ese modo, el trabajador quedó atrapado en la conducta, en el protocolo clínico, a sus patrones, manteniendo un lenguaje programado, sin posibilidad de nuevas interacciones, reduciendo el aspecto relacional de su trabajo, reduciendo su capacidad de percibir otras necesidades que no sean las dictadas por la ciencia.

Prosiguiendo, recurrimos una vez más a Merhy $(4,5)$, cuando afirma que la producción de la salud se realiza, sobre todo, a través del "trabajo vivo en acto", es decir, el trabajo humano en el exacto momento en que es ejecutado y que determina esa producción.

Ya vimos que el trabajo en salud se concretiza mediante los instrumentos, saberes, normas, máquinas; o sea, es un proceso de trabajo en el cual interactúan diversos tipos de tecnologías. Son esas interacciones que confieren un cierto sentido al modo de producir salud.

El trabajo hecho en acto es denominado "trabajo vivo en acto" y el trabajo realizado anteriormente, materializado como producto, es el "trabajo muerto" $(4,5,26)$. Merhy $(27)$ indica que en el trabajo coexisten los dos tipos de trabajo.

Así, en todo proceso de trabajo hay un ajuste del trabajo en acto (exacto momento de la actividad productiva) y el consumo de productos hechos en trabajos anteriores. Por ejemplo, la producción de un automóvil utiliza placas de acero; en el proceso de montaje en acto, los trabajadores utilizarán placas que Ilegaron listas a la industria automotriz. A su vez, el acero es producto de la producción siderúrgica, realizada anteriormente por otros trabajadores de la cadena metalmecánica. De esta forma, el trabajo de producir automóviles combina trabajos en acto de trabajadores que están utilizando el producto del trabajo antes realizado por otros trabajadores. Vale recordar que el trabajo vivo interactúa constantemente con el trabajo muerto, en procesos en los cuales interactúan diversos tipos de tecnologías. Por lo tanto, si todo trabajo es mediado por tecnologías, dependiendo de cómo ellas se combinan, puede haber procesos más creativos, centrados en las relaciones, o procesos más presos a la lógica de los instrumentos duros, como las máquinas.

En el desarrollo del proceso de trabajo en el área de la salud, que combina trabajo vivo y muerto, podemos observar que el comando -por trabajo vivo o muerto- se define dependiendo de la tecnología asumida por el profesional como referencia en la producción de la salud.

En el caso de Feliz, existe trabajo vivo y trabajo muerto en las dos situaciones de cuidado: de la cuidadora y del equipo. En el cuidado de la madre, prevalece una lógica no tecnificada de actuación: los actos de habla, escucha y la mirada. Las tecnologías blandas comandan el proceso de trabajo y es el trabajo vivo el que captura al muerto.

En el proceso de trabajo comandado por el trabajo vivo, la cuidadora detenta un gran margen de libertad para ser creativa, relacionarse con Feliz, experimentar soluciones para los problemas que surgen y -lo más importante- interactuar, incorporar al usuario en el proceso de producción de su misma salud, posibilitando que sea protagonista de su proceso salud-enfermedad (18). 
Tal perspectiva es central, tanto en la producción del cuidado individual de la salud de las personas como en la producción colectiva. Pues la participación activa de la comunidad en la toma de decisión sobre el ambiente en que vive es tan importante como la participación del usuario en la decisión sobre la mejor conducta clínica para su salud.

En la otra situación, el proceso de cuidado desarrollado por los profesionales es guiado por la tecnología dura (equipamientos y protocolos clínicos), llevando a la captura del trabajo vivo por el muerto, o sea, la acción del trabajador está presa al saber estructurado y al equipamiento, producto de un trabajo anterior. En este sentido, el proceso de trabajo es pre-programado, limitando la acción del trabajador a lo que ya fue determinado, por ejemplo, por la programación del protocolo clínico.

Esas orientaciones distintas para el plan de cuidado llevan a que se configure un cotidiano de disputas constantes entre la cuidadora y el equipo. La existencia de disputa no presupone necesariamente conflicto. $\mathrm{Al}$ contrario, puede propiciar mejoras en el proceso de trabajo desarrollado por el equipo, mientras exista una predisposición para entender, valorar, respetar al otro $y$, al mismo tiempo, posibilitar el entendimiento, valoración y respeto por el otro. Si cada profesional se dispone a mirar la situación de este modo, se permitirá afectar y ser afectado por el otro en su pensamiento, sentimiento y acción.

Durante las entrevistas con los profesionales involucrados en este caso, no se ha evidenciado esta disposición de escucha o aprendizaje. Al contrario, quedó claro que el equipo intenta someter a la cuidadora, buscando que cumpla el plan de cuidado elaborado por ellos, o bien intenta excluir/someter a la madre a través de algunas actitudes negativas, no compartiendo informaciones, ridiculizando su trabajo, etc.

Por otro lado, la cuidadora asume también el quiebre del diálogo, cuando ignora las recomendaciones del equipo, o cuando utiliza su papel de madre para obligar al equipo a aceptar algunas situaciones.

Queda claro, entonces, que la existencia de una diversidad de sujetos involucrados en la gestión del cuidado propicia constantemente arenas de disputas de ideas/proyectos individuales y/o colectivos para la orientación del trabajo. En este caso particular, esa arena de disputas se presenta como un problema para ambas partes.

Según Merhy (28), la institución de "plazas de conversación" entre los trabajadores y la cuidadora podría ser una forma de tornar el proceso más sinérgico, pues podría posibilitar el entendimiento del otro, propiciar pautas en la producción del cuidado y revelar otros asuntos pertinentes al usuario, al cuidador y al equipo.

El autor considera las "plazas" como espacios de comunicación donde los profesionales y las personas involucradas pueden exponer sus proyectos, deseos, necesidades frente a determinado asunto pertinente a la producción de la salud.

La expansión de la dimensión cuidadora podría ser otra manera de intentar aproximar más al equipo de la cuidadora. ¿Y cómo podría pasar eso?

Según Merhy (22), todo trabajador de salud, de una forma o de otra, produce cuidado, sea cuidado individual o colectivo. Hasta los profesionales no considerados propios de la salud, como los recepcionistas, pueden producir cuidado o des-cuidado.

Así, se cree que:

$\rightarrow$ los usuarios buscan en los encuentros con los trabajadores de salud, particularmente en los establecimientos de salud, la producción de espacios de protección, responsabilización y vínculo;

$\rightarrow$ el trabajo en salud no se limita al saber diagnosticar, pronosticar, curar, producir análisis epidemiológicos, realizar campañas, sino que también es un proceso y un espacio de producción de relaciones e intervenciones, que ocurre de modo compartido, y en el cual hay un juego entre necesidades y modos tecnológicos de actuar;

$\rightarrow$ no hay producción de espacios de intercambio, de hablar y escuchar, de complicidades y responsabilidades, de vínculos y aceptaciones, si no hay trabajo comprometido con la producción de valores de uso.

Mirando el caso desde estos conceptos, se puede decir que cada profesional y la cuidadora poseen su caja de herramientas con las tecnologías en salud, distribuidas en los núcleos de 
competencia específicos (tecnologías duras y blandas-duras) y en la dimensión cuidadora (tecnologías blandas). Lo que va a propiciar la distinción entre los miembros del equipo son las tecnologías que portan y la forma de operarlas, vinculada a los encuentros, a la historia de vida de cada uno, su cultura, condición social, etc.

Según Merhy, la dimensión cuidadora se revela como el territorio de las tecnologías blandas, no configurándose como campo específico de ninguna de las profesiones, sino como la base para la actuación de todos. Ella es el espacio de mayor intersección entre los trabajadores y entre trabajadores y usuarios, con un gran potencial para la producción del cuidado por parte del equipo, valorando los sentidos de la integralidad. Las tecnologías blandas, siendo utilizadas como las ordenadoras de la cadena productiva del cuidado, posibilitan un compromiso permanente del profesional, tales como vínculo-responsabilización, Ilevándolo a involucrarse con la resolutividad de la acción de salud (21) y con la garantía de autonomía del usuario, o sea, el usuario como protagonista de su proceso salud-enfermedad.

En el caso de Feliz, la interacción del equipo con la cuidadora se muestra difícil, pues la madre utiliza como guía las tecnologías blandas y el equipo las duras, disminuyendo el espacio de intercomunicación entre los dos campos de producción del cuidado. Además, los trabajadores no consideran la cuidadora como un miembro del equipo, dificultando aún más la producción de un cuidado más resolutivo para el niño.

\section{CONSIDERACIONES FINALES}

El trabajo en salud se produce mediante el encuentro (individual o colectivo) entre trabajadores y usuarios y se concreta por medio de la utilización de una combinación de tecnologías (duras, blandas-duras y blandas).

Ese encuentro involucra siempre un cierto grado de disputa entre planes de cuidado. Esa disputa se explicita más o menos dependiendo de las posibilidades de ejercicio de autonomía del usuario (o del cuidador en el caso de la atención domiciliaria). Además, dependiendo de la combinación de tecnologías y de la disposición de reconocimiento mutuo de los agentes, tiene como resultado el conflicto o la construcción compartida del plan de cuidado.

Para haber co-producción, es fundamental que el/los trabajador/es reconozcan como legítima la formulación de un plan de cuidado por parte del usuario o del cuidador y que las tecnologías que favorezcan la comunicación y la construcción de vínculos orienten el trabajo en salud.

En la atención domiciliaria existe una explicitación de la existencia de disputa de planes de cuidado, ya que uno de sus presupuestos es que el cuidador asuma parte de los cuidados que en otro escenario de práctica sería ofertada por los equipos de salud.

La figura del cuidador trae algunas tensiones y paradojas para el campo de la salud que necesitan ser mejor comprendidas, tanto en la cadena productiva del cuidado como en los efectos que ser cuidador produce sobre su misma salud y calidad de vida.

Se presenta -el cuidador- como un nuevo aliado, que puede contribuir al mejoramiento de la producción del cuidado, ya que trae las referencias del mundo de la vida hacia el escenario del trabajo en salud. 


\section{NOTAS FINALES}

a. El estudio analizó experiencias de Atención Domiciliaria en el ámbito del SUS en todas las regiones de país. Los servicios existentes en Sobral (Ce), Belo Horizonte (MG), Río de Janeiro $(\mathrm{RJ})$, Marilia (SP), Londrina (PR) han sido analizados de acuerdo con la metodología descripta.

b. "[...] son dispositivos inventados e implantados por los analistas institucionales para propiciar la explicitación de los conflictos y su resolución [...]" (10). c. Este nombre fue escogido por el hecho de que el niño demostraba constantemente este estado de espíritu, situación confirmada por los actores involucrados en el caso.

d. "[...] son los analizadores de hecho, producidos "espontáneamente" por la vida misma, históricosocial-libidinal y natural, como resultado de sus determinaciones y de su margen de libertad." (10).

\section{REFERENCIAS BIBLIOGRÁFICAS}

1. Donnangelo MCF. Saúde E Sociedade. San Pablo: Duas Cidades; 1976.

2. Gonçalves RBM. Tecnologia e organização social das práticas de saúde. San Pablo: Hucitec; 1994.

3. Luz MT. Natural, Racional, Social. Razón Médica y Racionalidad Científica Moderna. Buenos Aires: Lugar Editorial; 1997.

4. Merhy EE. Em busca do tempo perdido: A micropolítica do trabalho vivo em saúde. En:
Merhy EE, Onocko R, organizadores. Agir em saúde: um desafio para o público. San Pablo: Hucitec, Buenos Aires: Lugar Editorial; 1997.

5. Merhy EE. Salud: Cartografía del Trabajo Vivo. Buenos Aires: Lugar Editorial; 2006.

6. Pinheiro R, Ferla AA, Silva Junior AG. A integralidade na atenção à saúde da população. En: Marins JJN, Rego S, Lampert JB, Araújo JGC, organizadores. Educação médica em transformação: instrumentos para a construção de novas realidades. San Pablo: Hucitec, ABEM; 2004. p. 269-284.

7. Pinheiro R. As Práticas do Cotidiano na Relação Oferta e Demanda dos Serviços de 
Saúde: um campo de estudo e construção da integralidade. En: Pinheiro R, Mattos RA, organizadores. Os Sentidos da Integralidade na atenção e no cuidado à saúde. Río de Janeiro: IMS, UERJ, ABRASCO; 2001.

8. Franco TB, Merhy EE. Programa de Saúde da Família (PSF): Contradições de um Programa Destinado à Mudança do Modelo Tecnoassistencial. En: Merhy EE, et al. O trabalho em saúde: olhando e experienciando o SUS no cotidiano. San Pablo: Hucitec 2003. p. 55-124.

9. Ceccim RB. Equipe de Saúde: a perspectiva entre-disciplinar na produção dos atos terapêuticos. En: Pinheiro R, Mattos RA, organizadores. Cuidado: as fronteiras da integralidade. San Pablo: Hucitec; 2004.

10. Baremblit GF. Compêndio de Análise Institucional e outras correntes: teoria e prática. Río de Janeiro: Rosa dos Tempos; 1992.

11. Silva ALA. Produção de subjetividade e gestão em saúde: cartografias da gerência. [Tesis de Doctorado en Salud Colectiva]. Campinas: UNICAMP; 2004.

12. Kessner E, Kalk C, Singer J. Assessing Health Quality. The Case for Tracers. The New England Journal of Medicine. 1973;288(1):189-194.

13. Meneses CS. A unicidade do Sistema Único de Saúde em questão: a integralidade e a hierarquização do cuidado sob a perspectiva do trabalho vivo. [Disertación de Maestría en Salud Colectiva]. Campinas: UNICAMP; 1998.

14. Malta DC. Buscando novas modelagens em saúde, as contribuições do Projeto Vida e Acolhimento para a mudança do processo de trabalho na rede pública de Belo Horizonte, 1993-1996. [Tesis de Doctorado] Campinas: UNICAMP; 2001.

15. Jorge AO. A gestão hospitalar sob a perspectiva da micropolítica do trabalho vivo. [Tesis de Doctorado en Salud Colectiva]. Campinas: UNICAMP; 2002.

16. Silva Junior AG, Merhy EE, Carvalho LC. Refletindo sobre o ato de cuidar da saúde. En: Pinheiro R, Mattos RA, organizadores. Construção da integralidade: cotidiano, saberes e práticas em saúde. Río de Janeiro: IMS, UERJ; 2003. p. 89-112.

17. Franco TB. Fluxograma descritor e projetos terapêuticos para análise de serviços de saúde, em apoio ao planejamento: o caso de Luz (MG).
En: Merhy EE, et al., organizadores. O trabalho em saúde: olhando e experienciando o SUS no cotidiano. San Pablo: Hucitec; 2003. p. 161-198.

18. Ministério da Saúde. Curso de formação de facilitadores de educação permanente em saúde: unidade de aprendizagem-trabalho e relações na produção do cuidado em saúde. Río de Janeiro: Ministério da Saúde, Fiocruz; 2005.

19. Schiffler da Rocha AC, Campos Aguilera CE, Vieira Marques M; Mendonça PEX. Perspectivas da utilização do fluxograma analisador no ensino da administração em saúde na Faculdade de Medicina da UFRJ. Revista Brasileira de Educação Médica. 2005;29(3):191-200.

20. Cecílio de Oliveira LC. As necessidades de saúde como conceito estruturante na luta pela integralidade e equidade na atenção em saúde. En: Pinheiro R, Mattos RA, organizadores. Os Sentidos da Integralidade na atenção e no cuidado à saúde. Río de Janeiro: IMS, UERJ, ABRASCO; 2001.

21. Merhy EE. Em Busca da Qualidade dos Serviços de Saúde: os serviços de porta aberta para a saúde e o modelo tecnoassistencial em defesa da vida (ou como aproveitar os ruídos do cotidiano dos serviços de saúde e colegiadamente reorganizar o processo de trabalho na busca da qualidade das ações de saúde. En: Cecílio de Oliveira LC, organizador. Inventando a mudança na saúde. San Pablo: Hucitec; 1994.

22. Merhy EE. A perda da dimensão cuidadora na produção da saúde: uma discussão do modelo assistencial e da intervenção no seu modo de trabalhar a assistência. En: Campos CR, Malta DC, Reis AT, Santos AF, Merhy EE, organizadores. Sistema Único de Saúde em Belo Horizonte: reescrevendo o público. San Pablo: Xamã; 1998. p. 103-120.

23. Silva Junior AG. Lógicas de programas ações de saúde. En: Barboza P, organizador. Curso de Autogestão em Saúde. Río de Janeiro: Fiocruz, ENSP; 2001. p. 82-107.

24. Silva Junior AG, Mascarenhas MT. Avaliação da Atenção Básica em Saúde sob a Ótica da Integralidade Aspectos Conceituais e Metodológicos. En: Pinheiro R, Mattos RA, organizadores. Cuidado: as fronteiras da integralidade. San Pablo: Hucitec; 2004.

25. Merhy EE. O SUS e um dos seus dilemas: mudar a gestão e a lógica do processo de trabalho em saúde. En: Teixeira SF, organizadora. Movimento sanitário: 20 anos de democracia. San Pablo: CEBES; 1998. 
26. Franco TB. Processos de trabalho e transição tecnológica na saúde [Tesis de Doctorado]. Campinas: UNICAMP; 2003.

27. Merhy EE, Feuerwerker LCM. Atenção Domiciliar: lugar de encontro da medicalização com a rede substitutiva ou transição tecnológica e/ou reestruturação produtiva na saúde: um debate necessário na Atenção Domiciliar. Trabajo pre- sentado en el I Seminário Interno de Atenção Domiciliar da linha de pesquisa: Micropolítica do Trabalho e o Cuidado em Saúde. Río de Janeiro: UFRJ; 2006.

28. Merhy EE. Público e privado: entre aparelhos, rodas e praças. En: Aciole GG. A Saúde no Brasil: Cartografias do Público e do Privado. San Pablo: Hucitec; 2006.

\section{FORMA DE CITAR}

Carvalho LC, Feuerwerker LCM, Merhy EE. Disputas en torno a los planes de cuidado en la Internación Domiciliaria: una reflexión necesaria. Salud Colectiva. 2007;3(3):259-269.

Recibido el 5 de agosto de 2007

Versión final presentada el 23 de septiembre de 2007

Aprobado el 19 de octubre de 2007 